\title{
Evaluación del desempeño eléctrico de estructuras de diferentes materiales empleadas en redes de distribución
}

\section{Assessment of the electrical performance of power distribution structures composed by different materials}

\author{
Andrés Vanegas ${ }^{1}$, Esteban Velilla ${ }^{2}$, Alejandro Valencia ${ }^{3}$ \\ ${ }^{1}$ Unidad CET Normalización y Especificaciones, Empresas Públicas de Medellín, Colombia. Email: andres.vanegas@epm.com.co \\ ${ }^{2}$ Centro de Investigación, Innovación y Desarrollo de Materiales-CIDEMAT, Facultad de Ingeniería, Universidad de Antioquia, \\ Colombia. Email: esteban.velilla@udea.edu.co \\ ${ }^{3}$ Grupo de Manejo Eficiente de la Energía-GIMEL, Facultad de Ingeniería, Universidad de Antioquia, Colombia. \\ Email: jalejandro.valencia@udea.edu.co
}

ReCiBIDO: Abril 17, 2017. ACEPTADO: Agosto 10, 2017. Versión FinAL: Septiembre 30, 2017.

\begin{abstract}
RESUMEN
En este trabajo se evalúa el comportamiento eléctrico de estructuras considerando diferentes materiales conductivos y no conductivos en el sistema poste-cruceta. Para lograr este objetivo, se llevan a cabo ensayos tanto a frecuencia industrial como de impulso tipo descarga atmosférica para diferentes combinaciones de materiales que conforman una estructura típica empleada en el sistema de distribución de Empresas Públicas de Medellín (EPM). A partir de los resultados de los ensayos de tensión de impulso, es posible contrastar estos con los valores de referencia de la tensión de flameo al impulso crítico (CFO) establecidos en la norma IEEE Std. 1410. Finalmente, el comportamiento a frecuencia industrial es verificado por medio de simulaciones de campo electrostático usando el análisis de elementos finitos.
\end{abstract}

PALABRAS CLAVE: Postes; crucetas; redes de distribución; aislamiento; IEEE Std. 1410; elementos finitos.

\begin{abstract}
In this paper, the electrical behavior of structures is assessed considering conductive and non-conductive materials for a pole-crossarm arrangement. To achieve this, $\mathrm{AC}$ and impulse tests are performed for a typical distribution structure used in Empresas Públicas de Medellín (EPM) distribution system and composed by pole and crossarm for different combination of materials. From the result obtained by the impulse test, it is possible to stablish the contrast with the critical flash overvoltage (CFO) reference values published in the IEEE Std. 1410. Finally, the low-frequency behavior is verified by means electrostatic field simulation using the finite element analysis.
\end{abstract}

KEYWORDS: Utility poles; crossarms; distribution networks; insulation; IEEE Std. 1410; finite elements.

ISSN Impreso: 1657 - 4583, En Línea: 2145 - 8456

Este artículo puede compartirse bajo la licencia CC BY-ND 4.0 y se referencia usando el siguiente formato: A. Vanegas, E. Velilla, J. A. Valencia, "Evaluación del desempeño eléctrico de estructuras de diferentes materiales empleadas en redes de distribución," Rev. UIS Ing., vol. 17, no. 1, pp. 35-42, 2018. Doi: https://doi.org/10.18273/revuin.v17n1-2018003 


\section{INTRODUCCIÓN}

Las estructuras como postes y crucetas son esenciales para el soporte de las redes aéreas de distribución. En Colombia, los postes de madera, los postes de concreto, las crucetas de madera y las crucetas de acero, han sido los elementos estructurales estándar empleados por los operadores de red. Actualmente, y como una industria con demanda creciente, se encuentran alternativas como los postes de acero, los postes de poliéster reforzado con fibra de vidrio (PRFV) y las crucetas de PRFV. A pesar de que la función principal de los postes y crucetas es mecánica o estructural, las características dieléctricas de materiales como la madera o el PRFV pueden desempeñar un papel relevante en el diseño del aislamiento de la red y en particular, su comportamiento frente a sobretensiones originadas por descargas atmosféricas (DA) [1]. De esta manera, la norma IEEE Std 1410 [2] pretende asistir al diseño del aislamiento de una red de distribución (RD) y su protección frente a DA. Según esta norma, las RD pueden ser diseñadas teniendo en cuenta algunas estrategias, entre las cuales está considerar un mayor nivel de aislamiento, acorde con las condiciones locales de resistividades de los suelos, tal que la red pueda soportar las más severas sobretensiones inducidas [2], [3]. Un valor de aislamiento (CFO en húmedo) de $300 \mathrm{kV}$ es considerado un valor aceptable de aislamiento para la mayoría de RD con tensión nominal hasta $69 \mathrm{kV}$ [2], y ha sido referenciado en otras publicaciones como EPRI [4] y RUS [5]. Como se observa en la Tabla 1, estructuras de acero, no aportan al aislamiento de la red [6]. Por su parte, el valor de aislamiento (CFO) para aisladores empleados en RD de $13.2 \mathrm{kV}$ normalmente se encuentran entre 100 y $150 \mathrm{kV}$. De esta manera si en una estructura no se consideran elementos de madera o de PRFV, se tendría un valor de CFO inferior al recomendado por la norma IEEE Std 1410, puesto que el CFO total de la estructura estaría solamente representado por los aisladores.

Tabla 1. Comportamiento eléctrico de diferentes materiales mediante ensayos de flameo a frecuencia industrial y a tensión de impulso (CFO) bajo condiciones secas y húmedas.

\begin{tabular}{|c|c|c|c|c|}
\hline \multirow{2}{*}{$\begin{array}{c}\text { Material } \\
\text { de la } \\
\text { estructura }\end{array}$} & \multicolumn{3}{|c|}{ Comportamiento eléctrico } \\
\cline { 2 - 5 } & Frecuencia industrial & \multicolumn{2}{c|}{ CFO } \\
\hline Acero & Ceco & Húmedo & Seco & Húmedo \\
\hline Madera & Aislante & Conductivo & Aislante & Aislante \\
\hline PRFV & Aislante & Conductivo & Aislante & Aislante \\
\hline
\end{tabular}

Fuente. S. Grzybowski y T. Disyadej [6].

\section{DENSIDAD DE DESCARGAS ATMOSFÉRICAS Y BREVE REVISIÓN DE LA NORMA IEEE STD 1410}

El territorio colombiano se encuentra en una zona con un alto número descargas atmosféricas anuales, la Figura 1 muestra el mapa de la densidad de descargas atmosféricas a tierra (DDT) para el departamento de Antioquia obtenido para los años 2012 a 2014, donde se observan zonas críticas tales como el este y nordeste del departamento, donde la variable DDT puede superar las 30 descargas por $\mathrm{km}^{2}$ en el año. Con relación a la incidencia de las DA en las redes de media tensión, estas tienen un efecto negativo en la confiabilidad e índices de calidad de energía de un sistema eléctrico [2], [7]-[9]. En particular, para las redes de EPM, como muestra la Figura 2, el alto valor de la DDT en regiones del norte del departamento de Antioquia, ha traído inconvenientes en la operación y mantenimiento, reportándose continuas fallas en aisladores en días de tormenta. La norma IEEE Std. 1410 recomienda incorporar aislamientos complementarios usando materiales dieléctricos para las crucetas o postes con la finalidad de mejorar el desempeño de las RD ante las descargas atmosféricas. De esta manera, el CFO combinado donde dos o más componentes en serie involucrados en el aislamiento de la red, dado por la Ecuación (1), se estableció en la norma IEEE Std 1410.

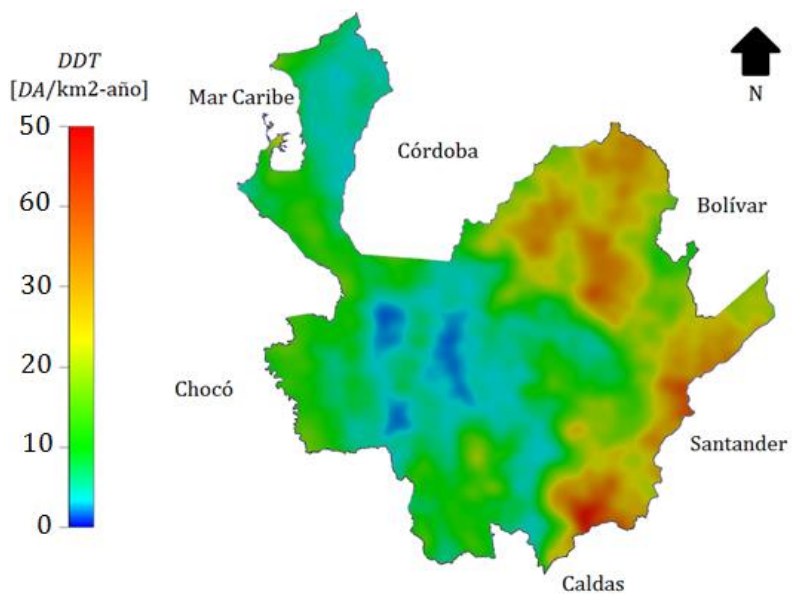

Figura 1. Mapa de DDT para el departamento de Antioquia, años 2012 - 2014. Fuente. KERAUNOS - SIATA. [10], [11]. 


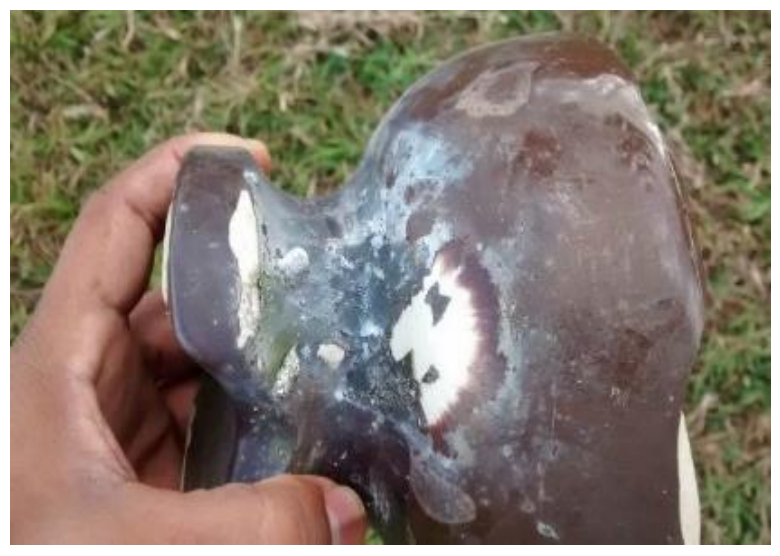

Figura 2. Falla de un aislador ANSI 55-4 causado por DA en $\mathrm{RD}$ de $13.2 \mathrm{kV}$. Fuente. Elaboración propia.

$$
C F O_{T}=C F O_{\text {aisl }}+C F O_{\text {adic. } 2^{\circ} \text { comp }}+C F O_{\text {adic. } .3^{\circ} \text { comp }}+\cdots C F O_{\text {adic. } n^{\circ} \text { comp }}
$$

Los términos de la Ecuación (1) cuando se adicionan elementos estructurales como postes y crucetas de madera o PRFV a un aislamiento primario dado por un aislador ANSI 55-4, se presentan en la Tabla 2. Según la norma IEEE Std 1410, los valores de CFO de la Tabla 2 son obtenidos mediante ensayos normalizados en húmedo, igualmente, estos hacen referencia al valor mínimo obtenido entre impulsos de polaridad positiva y negativa. Esta norma informa que los valores de CFO de componentes adicionados al aislamiento primario tienen un $20 \%$ de error, así, dada la variedad de materias primas empleadas para la construcción de crucetas y postes de madera o de PRFV, la norma recomienda realizar ensayos de laboratorio para determinar con mayor exactitud estos valores.

Tabla 2. CFO de componentes en serie con un aislador ANSI 55-4.

\begin{tabular}{|c|c|c|c|}
\hline Comp & $\begin{array}{c}C F \\
O \\
\text { aisl } \dagger\end{array}$ & Comp. $\left(\mathrm{CFO}_{\text {adic }^{\circ}{ }^{\circ} \text { comp }}\right) \ddagger$ & $\begin{array}{c}\text { Comp. } \mathbf{y} \\
\text { CFO }_{\text {adic } 3^{\circ} \text { comp }}\end{array}$ \\
\hline \multirow{4}{*}{ 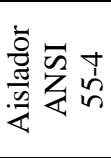 } & \multirow{4}{*}{$\begin{array}{l}105 \\
\mathrm{kV}\end{array}$} & Poste madera $(235 \mathrm{kV} / \mathrm{m})$ & \multirow{4}{*}{$\begin{array}{c}\text { Poste madera } \\
(65 \mathrm{kV} / \mathrm{m}) \\
\text { Poste PRFV } \\
(94 \mathrm{kV} / \mathrm{m})\end{array}$} \\
\hline & & Cruceta madera $(250 \mathrm{kV} / \mathrm{m})$ & \\
\hline & & Poste PRFV (400 kV/m) & \\
\hline & & Cruceta PRFV $(250 \mathrm{kV} / \mathrm{m})$ & \\
\hline
\end{tabular}

$\uparrow \mathrm{CFO}_{\text {aisl }}$ : $\mathrm{CFO}$ del aislador (componente primario)

CFO ${\text { adic } 2^{\circ} \text { comp }}$ : $\mathrm{CFO}$ adicionado por el segundo componente

$+C F O_{\text {adic } 3{ }^{\circ} \text { comp }}$ : $\mathrm{CFO}$ adicionado por el tercer componente

Fuente. IEEE Std. 1410 [2].

\section{ENSAYOS A FRECUENCIA INDUSTRIAL}

Para estos ensayos se empleó una fuente Phenix Technologies BK130, propiedad del laboratorio EATIC de EPM, la cual proporciona hasta $130 \mathrm{kV}$ y una corriente máxima de $50 \mathrm{~mA}$ a $60 \mathrm{~Hz}$. Se seleccionó una estructura de frecuente uso en las RD de $13.2 \mathrm{kV}$ de EPM, conocida, según su norma de construcción, como RA2-001 [12], la cual emplea una cruceta de $1500 \mathrm{~mm}$ de longitud total e instalada en el poste mediante una diagonal metálica. La conexión de la fuente y el objeto de prueba, se realizó en los extremos (1) y (2), como muestra la Figura 3, el objeto de prueba consistió en la combinación de postes de concreto, acero y PRFV con crucetas de acero, madera y PRFV, un tubo de aluminio 5/8" se utilizó para simular el conductor, soportado por un aislador ANSI 55-4; la tasa de creciemiento de la tensión fue de $1 \mathrm{kV}$ por segundo, de acuerdo con las recomendaciones establecidas en la norma IEEE Std 4 [13].

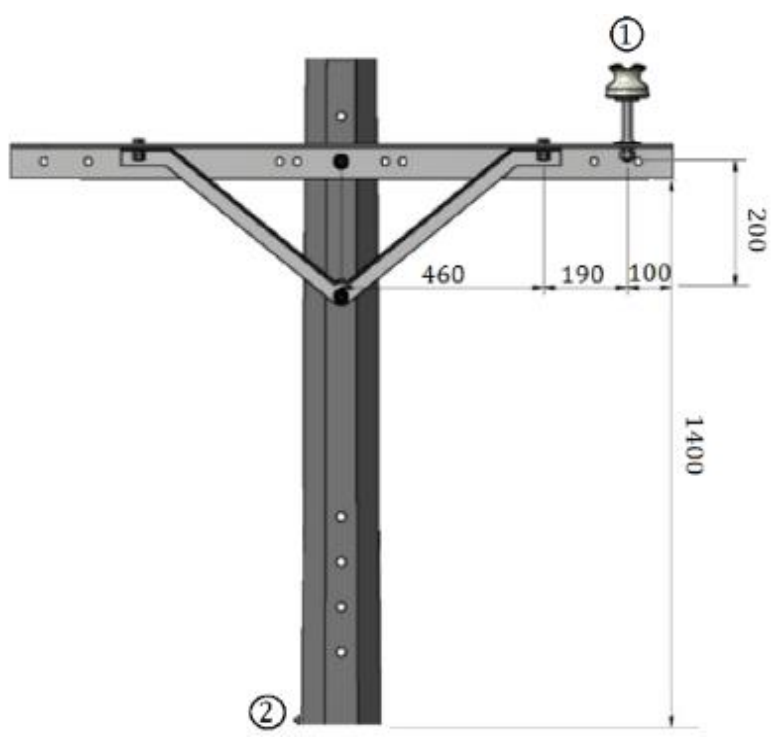

Figura 3. Objeto de prueba, basado en la estructura RA2-001 [12]. Dimensiones en mm. Fuente. Elaboración propia.

En particular, la cruceta de PRFV, como se muestra en la Figura 4 puede ser empleada sin el uso de diagonales metálicas, por esta razón, en los ensayos con cruceta de fibra de vidrio, se incluye esta variante. Por su parte, para los casos con cruceta de madera, el montaje solo fue considerado sin diagonales metálicas. 


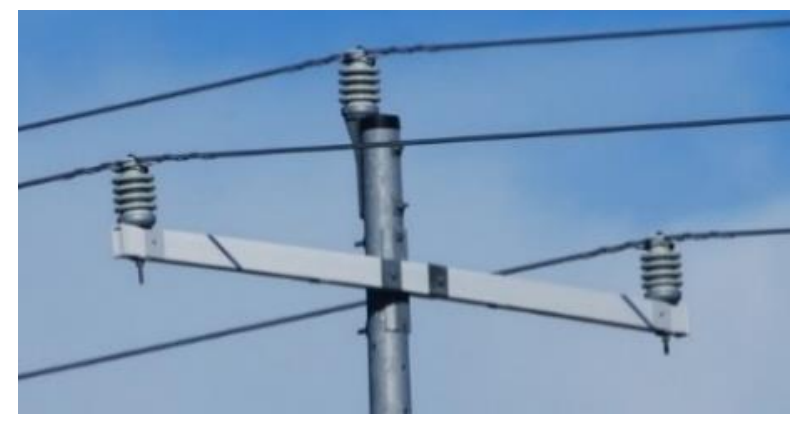

Figura 4. Cruceta de PRFV sin diagonales metálicas. Fuente. PUPI Crossarms [14].

\subsection{Resultados}

En la Tabla 3, se muestra el promedio de diez ensayos para condiciones secas y húmedas, el valor reportado es el valor de la tensión que causa el flameo en el $50 \%$ de aplicaciones, para las diferentes composiciones de la estructura. Los valores son corregidos a condiciones ambientales estándar, teniendo en cuenta que los ensayos se realizaron a una altura de 1482 metros sobre el nivel del mar. Las pruebas en húmedo se realizaron con un aspersor de 36 agujeros de $0.5 \mathrm{~mm}$ de diámetro.

Tabla 3. Tensión de flameo a frecuencia industrial para diferentes combinaciones de materiales para el sistema postecruceta.

\begin{tabular}{|c|c|c|c|}
\hline \multicolumn{2}{|c|}{ Composición de la estructura } & \multirow{2}{*}{$\begin{array}{c}\text { Tensión } \\
\text { de flameo } \\
\text { (kV rms) } \\
\text { en seco }\end{array}$} & \multirow{2}{*}{$\begin{array}{c}\text { Tensión de } \\
\text { flameo (kV } \\
\text { rms) en } \\
\text { húmedo }\end{array}$} \\
\hline $\begin{array}{c}\text { Poste } \\
\left(\mathrm{T}_{\text {prom. }}\right) \\
{\left[\mathrm{Hr}_{\text {prom. }}\right]}\end{array}$ & Cruceta & & \\
\hline \multirow{4}{*}{$\begin{array}{c}\text { Concreto } \\
\left(29.3^{\circ} \mathrm{C}\right) \\
{[38.1 \%]}\end{array}$} & Acero con diagonales & 68.24 & 40.98 \\
\hline & Madera sin diagonales & 102.89 & 57.17 \\
\hline & PRFV con diagonales & 91.19 & $50.23 \div$ \\
\hline & PRFV sin diagonales & $>130 \dagger$ & 86.18 \\
\hline \multirow{4}{*}{$\begin{array}{c}\text { Acero } \\
\left(28.6^{\circ} \mathrm{C}\right) \\
{[40.3 \%]}\end{array}$} & Acero con diagonales & 75.53 & 35.17 \\
\hline & Madera sin diagonales & 96.95 & 42.36 \\
\hline & PRFV con diagonales & 95.75 & $47.93+$ \\
\hline & PRFV sin diagonales & $>130 \dagger$ & 94.13 \\
\hline $\begin{array}{c}\text { PRFV } \\
\left(25.2^{\circ} \mathrm{C}\right) \\
{[47.2 \%]}\end{array}$ & Acero con diagonales & $>130 \dagger$ & $>130 \dagger$ \\
\hline
\end{tabular}

$\uparrow$ Valor máximo sin ocurrencia de flameo.

$\ddagger$ Pruebas destructivas, sin suficientes datos para obtener un promedio confiable de ensayos.

$\mathrm{T}_{\text {prom.: }}$ : temperatura promedio del ensayo.

$\mathrm{Hr}_{\text {prom}}$ : humedad relativa promedio del ensayo.

Fuente. Elaboración propia.

Como se aprecia en la Tabla 3, la condición más desfavorable desde el punto de vista de aislamiento, se presenta en los casos de estructuras totalmente conductivas (postes de concreto o acero, con cruceta de acero), los flameos registrados en condiciones secas de $75.53 \mathrm{kV}$ y $68.24 \mathrm{kV}$, para el uso de poste de acero y de concreto respectivamente y sus correspondientes en condiciones húmedas, se presentan siguiendo la distancia de arco del aislador, como ejemplo, la Figura 5 muestra uno de los flameos en seco registrados con la estructura compuesta por poste y cruceta de acero.

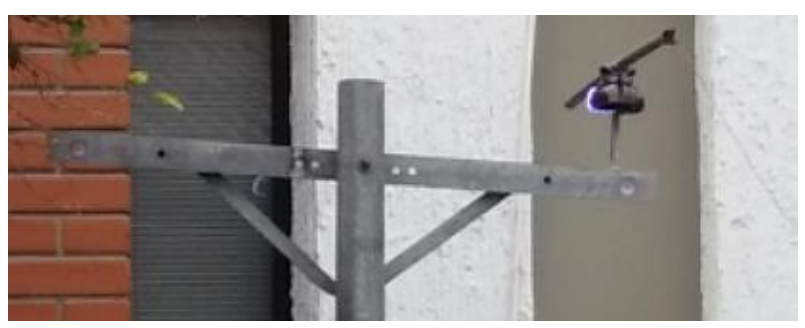

Figura 5. Flameo en seco. Estructura compuesta por poste y cruceta de acero. Fuente. Elaboración propia.

Para el caso de la estructura compuesta por poste conductivo y cruceta de PRFV con diagonales metálicas, como se observa en la Figura 6, el flameo en seco se presenta entre el conductor y el tornillo de fijación de la diagonal, que comprende una distancia de $200 \mathrm{~mm}$ aproximadamente. De esta manera, el flameo en seco obtenido fue de $95.75 \mathrm{kV}$ y $91.19 \mathrm{kV}$ para las estructuras con poste de acero y concreto, respectivamente.

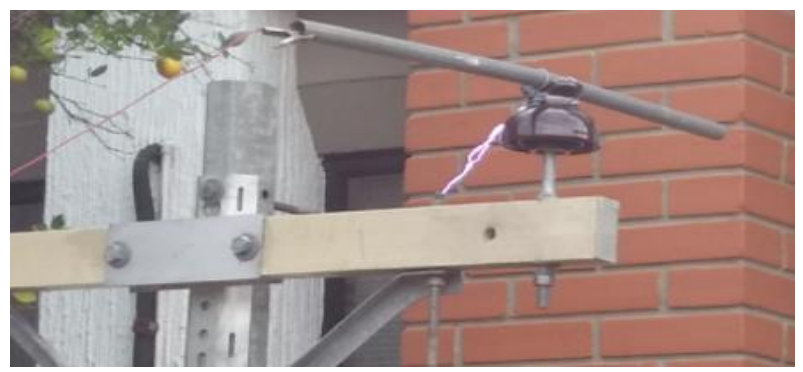

Figura 6. Flameo en seco. Estructura compuesta por poste y cruceta de PRFV con diagonales metálicas. Fuente. Elaboración propia.

Para los flameos en húmedo, en los casos cuando se emplean crucetas de PRFV con diagonales metálicas, la corriente de fuga del ensayo causó falla del material por quemadura del mismo, de esta manera, el valor de tensión alcanzado en la configuración con poste de acero fue de $57.9 \mathrm{kV}$ antes de que la cruceta comenzara a quemarse. Por su parte, para el caso con poste de concreto, el valor alcanzado fue de $50.23 \mathrm{kV}$. Para la condición de instalación de cruceta de PRFV sin diagonales metálicas, en el ensayo en condiciones secas, las configuraciones empleando postes de concreto y de acero, superaron los $130 \mathrm{kV}$ sin presentar flameo o afectaciones del material. Sin embargo, para condiciones húmedas, el material 
presentó afectación a tensiones de $94.13 \mathrm{kV}$ y $86.18 \mathrm{kV}$ para las estructuras con poste de acero y de concreto, respectivamente. Adicionalmente se observa un mejor comportamiento de las crucetas de PRFV frente a las crucetas de madera. Para finalizar, se observa que la condición más favorable para el aislamiento de la red, se presenta mediante el uso de postes de PRFV, en estos ensayos, se observa que se supera el valor de $130 \mathrm{kV}$ sin presencia de flameo o afectación del material.

\section{ENSAYOS A TENSIÓN DE IMPULSO}

Los ensayos de impulso tipo DA se llevaron a cabo en el laboratorio de alta tensión del fabricante de transformadores Rymel [15], se empleó un generador de impulsos con una capacidad máxima de tensión pico de $420 \mathrm{kV}$ de onda doble exponencial $(1.2 / 50 \mu \mathrm{s})$ [13], la conexión entre el generador de impulso y el objeto de prueba, ubicado entre los extremos (1) y (2), fue idéntica a la mostrada en la la Figura 3. A diferencia de los ensayos a frecuencia industrial, en los ensayos a impulsos de tensión, no se consideraron casos con postes de concreto ni con crucetas de madera, del mismo modo, no se realizaron pruebas en húmedo.

\subsection{Resultados}

En Tabla 4, se muestran los resultados de la tensión pico de la onda de impulso, de polaridad negativa, que causa flameo en el $50 \%$ de aplicaciones (CFO en condiciones secas), para las diferentes composiciones de la estructura. Los valores de CFO son obtenidos mediante el método up-and-down [13], y corregidos a condiciones ambientales estándar, teniendo en cuenta que los ensayos se realizaron a una altura de 1425 metros sobre el nivel del mar.

Tabla 4. CFO en condiciones secas, mediante impulsos negativos de onda 1.2/50 $\mu$ s. corregido a condiciones estándar.

\begin{tabular}{|c|c|c|}
\hline \multicolumn{2}{|c|}{ Composición de la estructura } & \multirow{2}{*}{$\begin{array}{c}\text { CFO } \\
\left(\mathrm{kV}_{\text {pico }}\right) \\
\text { en seco }\end{array}$} \\
\hline $\begin{array}{c}\text { Poste (T Prom.) [Hr } \\
\text { Prom.] }\end{array}$ & Cruceta & \\
\hline \multirow{3}{*}{ Acero $\left(22.3^{\circ} \mathrm{C}\right)[80 \%]$} & Acero & 151.9 \\
\hline & PRFV con diagonales & 170.4 \\
\hline & PRFV sin diagonales & $345.6 \mathrm{kV}$ \\
\hline $\operatorname{PRFV}\left(24^{\circ} \mathrm{C}\right)[73 \%]$ & Acero & $>420 \dagger$ \\
\hline
\end{tabular}

$\dagger$ Valor máximo sin ocurrencia de flameo.

Fuente. Elaboración propia.

En la Figura 7, se muestran los flameos registrados para las diferentes combinaciones de poste de acero con cruceta de acero y con cruceta de PRFV, con y sin diagonales metálicas. Se observa que el camino de del flameo es similar al flameo en condiciones secas a frecuencia industrial, si se compara la Figura 7(a) y (b), con lo presentado en las Figuras 5 y 6 . Adicionalmente, la Figura 7(c) muestra el flameo entre el conductor y el soporte metálico de la cruceta sin diagonales.

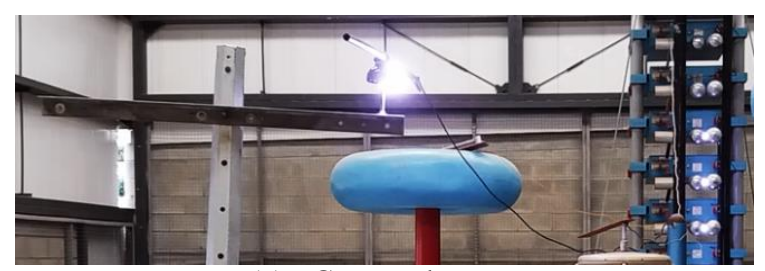

(a) Cruceta de acero

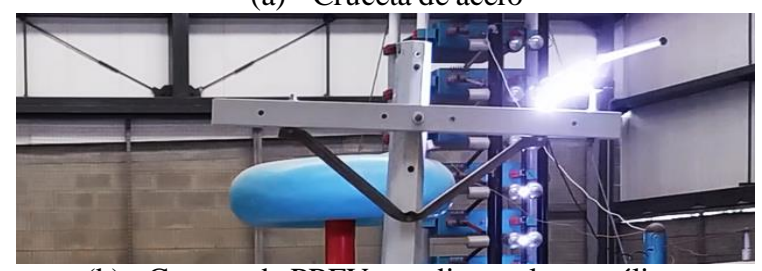

(b) Cruceta de PRFV con diagonales metálicas

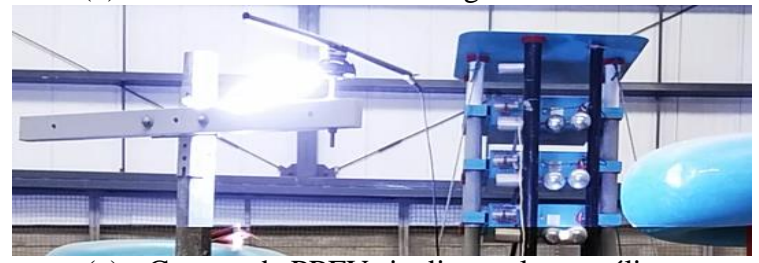

(c) Cruceta de PRFV sin diagonales metálicas

Figura 7. Flameos en seco para diferentes configuraciones de crucetas empleando poste de acero. Fuente. Elaboración propia.

Si los valores de flameo de la Tabla 4 son afectados por el factor de $0.8 \pm 0.1$, recomendado por la guía IEEE Std 1410 para obtener el valor de CFO en condiciones húmedas, se concluye que ninguna de las configuraciones con poste de acero, cumple con el valor de referencia de $300 \mathrm{kV}$ de CFO, establecido por la norma IEEE Std 410. Como ejemplo, el valor de $345.6 \mathrm{kV}$ obtenido para la configuración de cruceta de fibra de vidrio sin diagonales, es afectado por el factor mencionado, resulta en $276.5 \mathrm{kV}$ con un error de $\pm 13 \%$. Con el objetivo de comprobar la Ecuación (1) para la configuración de cruceta de PRFV sin diagonales con poste de acero, se emplean los valores de la Tabla 3 , y teniendo en cuenta la longitud de $0.6 \mathrm{~m}$ sobre la cruceta de PRFV, medida entre el punto de fijación del aislador y el soporte metálico de la cructa para su fijación al poste, se obtiene el valor de CFO de $255 \mathrm{kV}$ dado por la Ecuación (2).

$$
\begin{aligned}
& C F O_{T}=C F O_{\text {aisl }}+C F O_{{\text {adic } 2^{\circ} \text { comp }}} \\
& C F O_{T}=105 \mathrm{kV}+250 \mathrm{kV} / \mathrm{m} \cdot(0.6 \mathrm{~m})=255 \mathrm{kV}
\end{aligned}
$$


Se concluye que esta estructura requiere aumentar la distancia de la cruceta de PRFV, para cumplir las recomendaciones de la IEEE Std 1410.

\section{EVALUACIÓN POR ELEMENTOS FINITOS DE CAMPOS ELECTROSTÁTICOS}

El análisis por elementos finitos aplicado a casos de evaluación de aislamiento y distancias en estrucutras de redes de distribución, ha sido presentado previamente por Biasotto et al [16], [17]. El programa Simulation Mechanical [18] es empleado en este trabajo para representar e ilustrar el uso de estructuras con diferentes características eléctricas. Se parte de un modelo CAD en 2D mostrado en la Figura 8(a), el cual será utilizado para representar la geometría de la estrucutra y se conservan las mismas dimensiones empleadas en las estructuras de los ensayos de las secciones anteriores e iustrado en la Figura 3. A continuación, en la Figura 8(b) se muestra la discretización empleada, la permitividad relativa de la porcelana, $\varepsilon r$, se consideró igual 5.5 , valor tomado como como referencia a $25^{\circ} \mathrm{C}$ y a baja frecuencia $(<1 \mathrm{kHz})$. Del mismo modo, se consideró el aire con $\varepsilon r=1$.

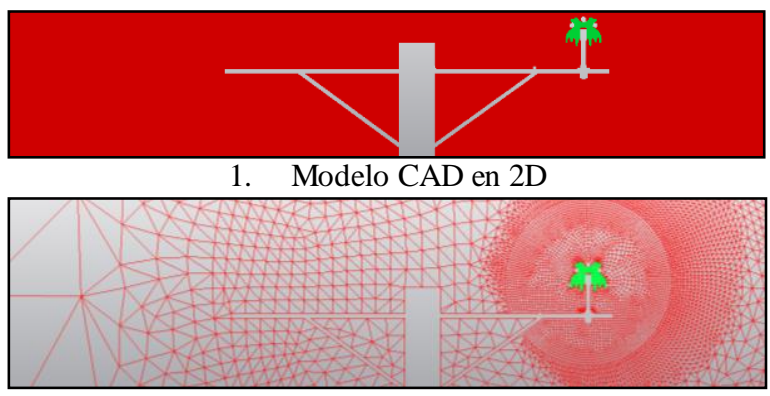

2. Discretización de elementos finitos de la estructura

Figura 8. Modelos para la evaluación computacional de campos electrostáticos. Fuente. Elaboración propia.

\subsection{Caso I: estructura conductiva}

En este caso, la estructura es puesta a un potencial de $0 \mathrm{~V}$, dado que es considerada de material conductivo, compuesta por un poste de concreto o de acero, por cruceta y diagonal de acero y por un espigo metálico. En la Figura 9(a) se muestra la distribución de potencial generado en condiciones normales de operación cuando la tensión en el conductor es $10.78 \mathrm{kV}$, que corresponde al valor pico del valor monofásico nominal de una RD de $13.2 \mathrm{kV}$. Adicionalmente, la Figura 9 muestra la distribución de potencial cuando la tensión en el conductor alcanza el valor de $96.51 \mathrm{kV}$ pico $(68.24 \mathrm{kV}$ rms), valor tomado de la Tabla 3 registrado en el ensayo de flameo a baja frecuencia para la configuración de poste de concreto y cruceta de acero. El camino de flameo que se presenta en una estructura conductiva es el mostrado en la Figura 5, esto es, siguiendo la distancia de arco del aislador (entre el conductor y el espigo). Puede observarse que la línea equipotencial más cerca del espigo, cambia de $714 \mathrm{~V}$ a $3490 \mathrm{~V}$ en las dos condiciones de la Figura 9.

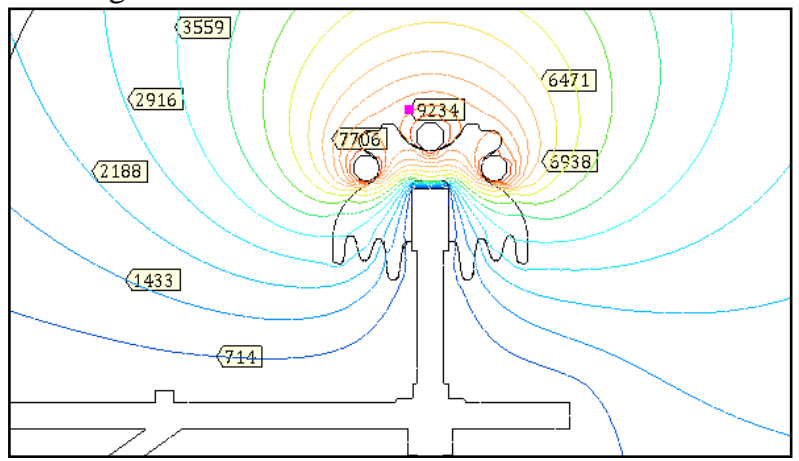

(a) Condiciones normales de operación

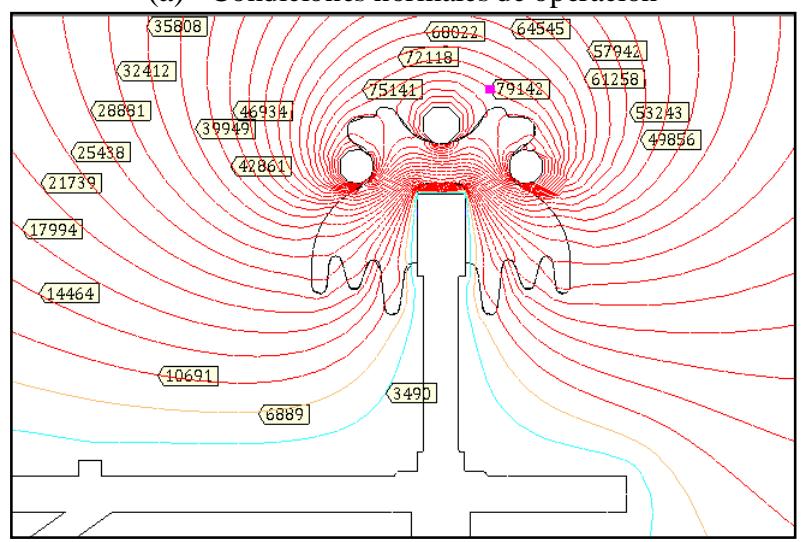

(b) Condición de flameo

Figura 9. Equipotenciales para una estructura conductiva. Fuente. Elaboración propia.

\subsection{Caso II: estructura con poste conductivo y una cruceta de PRFV con diagonal metálica}

En este caso las diagonales y el poste son puestos a $0 \mathrm{~V}$, adicionalmente, la permitividad del material de la cruceta se toma igual a 5.2, valor de referencia, de acuerdo con el manual de perfiles de PRFV del fabricante de crucetas Creative Pultrusions [19]. Con las anteriores consideraciones, Figura 10(a) y (b) muestra las distribuciones de potencial para las condiciones nominales de operación y para la condición de flameo, respectivamente. Para la condición de flameo se empleó una tensión de $128.96 \mathrm{kV}$ pico $(91.19 \mathrm{kV}$ rms), valor tomado de la Tabla 3, registrado en el ensayo de flameo. El camino de flameo que se presenta en un este caso es el mostrado en la Figura 6, esto es, siguiendo la distancia entre el aislador y el tornillo de fijación de la diagonal a la cruceta. Puede observarse que la línea equipotencial 
más cerca del tornillo, cambia de $1092 \mathrm{~V}$ (Figura 10(a)) a 3332 V (Figura 10(b)).

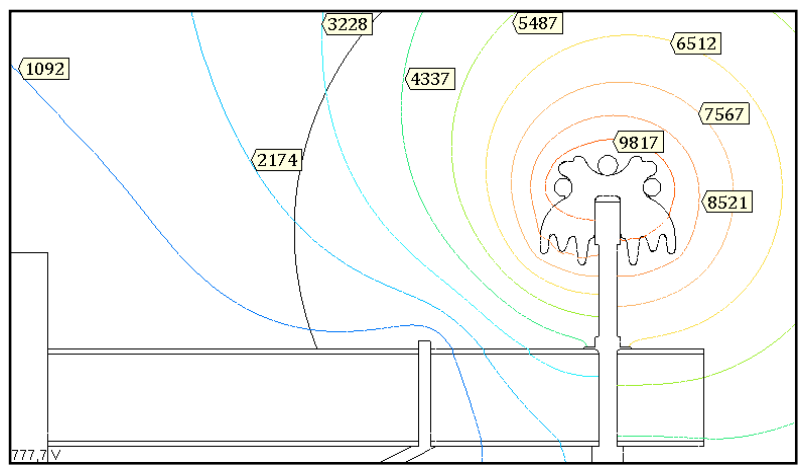

(a) Condiciones normales de operación

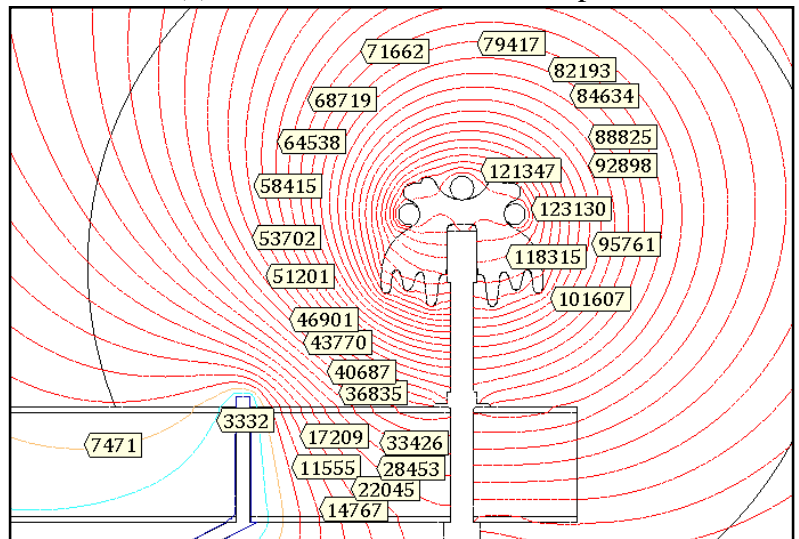

(b) Condiciones normales de operación

Figura 10. Equipotenciales para un poste conductivo y una crucera de PRFV con diagonal metálica. Fuente. Elaboración propia.

\section{CONCLUSIONES}

Debido a la alta densidad de DA de algunas zonas del territorio colombiano, las estructuras de madera o de PRFV, a diferencia de las construidas con materiales como el acero o el concreto, son alternativas importantes para mejorar el desempeño del aislamiento de las RD. De esta manera, la norma IEEE Std 1410 debe ser valorada en el diseño del aislamiento por los operadores de red en Colombia.

En los ensayos realizados, se observa que el PRFV tiene el mejor desempeño dentro los materiales evaluados para ser considerado como aislamiento complementario. En los ensayos de flameo a frecuencia industrial, aunque el desempeño en condiciones secas de la cruceta de madera presenta un comportamiento aceptable, su retención de agua en las pruebas con condiciones húmedas, hace que el desempeño de su aislamiento disminuya considerablemente. Por su parte, como consecuencia de los ensayos de flameo a tensión de impulso y de la revisón de la norma IEEE Std 1410, se concluye que el uso de configuraciones con un poste de material conductivo (acero o concreto) en conjunto con crucetas de $1500 \mathrm{~mm}$ de longitud de PRFV, debe ser replanteada puesto que presenta un valor de aislamiento (CFO) inferior a $300 \mathrm{kV}$; esto puede ser solucionado, aumentado la longitud de las crucetas de este material. Análisis similares a los presentados al final de la sección 4, en combinación con ensayos a tensión de impulso, para determinar el CFO combinado de aislamientos en serie, pueden realizarse para cualquier otra configuración con el objetivo de cumplir con las recomendaciones de la IEEE Std 1410.

Finalmente, el apoyo por medio de simulación con elementos finitos representa la distribución de potenciales a frecuencia industrial, así las zonas de la estructura con probabilidades altas de flameo ante aumentos de la tensión da la red pueden ser detectados, de esta manera se determina que lineas equipotenciales con valores cercanos a $3300 \mathrm{~V}$ en proximidades de elementos conectados a tierra, presentan probabilidad de flameo en los casos evaluados en la sección 5.

\section{REFERENCIAS}

[1] A. Vanegas. "Evaluación computacional y experimental del desempeño mecánico y eléctrico de estructuras usadas en redes de distribución de energía eléctrica". Facultad de Ingeniería. Trabajo de Investigación de Maestría: Universidad de Antioquia. Medellín, Colombia, 2017.

[2] IEEE Guide for Improving the lightning Performance of Distribution Lines. IEEE Std 1410, 2010.

[3] A. Vanegas, E. Velilla, J. Herrera, and J. A. Valencia, "An efficient procedure for computing lightning induced overvoltages on overhead lines," CPE 2009 - 6th Intern. Conf.-Wshp.-Compatibility and Power Electro., 2009, pp. 368-374.

[4] Electric Power Research Inst., "Handbook for Improving Overhead Transmission Line Lightning Performance," 2004.

[5] United States Dept. of Agr., RUS - Guidelines and Approval for the Use of Steel Distribution Poles, July 2008, pp. 1-6.

[6] S. Grzybowski and T. Disyadej, "Electrical performance of fiberglass crossarm in distribution and transmission lines," Transm. Distrib. Expo. Conf. 2008 
IEEE PES Powering Towar. Futur. PIMS 2008, pp. 1-5, 2008.

[7] Keraunos S.A.S, CREG, "Identificación y análisis de los niveles de calidad del servicio alcanzables en las redes de distribución de energía eléctrica del SIN," Bogotá, 2013.

[8] L. E. Perdomo O., A. Rodríguez, F. Santamaría. "Metodología para el registro de parámetros de calidad de energía en microrredes Inteligentes," Rev. UIS Ing., vol. 15, no. 2, pp. 117-123, 2016.

[9] M. A. Ángel S., G. Ordoñez, "Calidad de la energía eléctrica: diseño y construcción de un prototipo como alternativa para la monitorización de interrupciones y caídas de tensión," Rev. UIS Ing., vol. 4, no. 2, pp. 75-86, 2011.

[10] Keraunos S.A.S. [En Línea] Disponible en: http://keraunos.co/index. php/es-co/

[11] "Sist. de Alerta Temprana de Medellín y el Valle de Aburrá -SIATA." [En Línea] Disponible en: https://siata.gov.co/sitio_web/index.

php.

[12] EPM, "RA2-001," Empresas Públicas de Medellín, E.S.P., 2013. [En Línea] Disponible en: http://www.epm.com.co/site/portals/7/Documentos/RA2 -001.pdf

[13]IEEE Standard for High-Voltage Testing Techniques. IEEE Std 4, 2013.

[14]Geotek Inc., "PUPI All Aplication Photos." [En Línea] Disponible en: http://www.pupicrossarms.com/Products/All_Applicatio n_Photos.php.

[15]"Rymel S.A.S.”. [En Línea] Disponible en: http://www.rymel.com.co/

[16] P. S. De Oliveira, E. Biasotto, M. A. A. Lima, J. C. M. Vieira, and M. Oleskovicz, "Voltage Elevation Analysis of Overhead Distribution Lines Using the Finite Element Method," in Power and Energy Society General Meeting, 2011 IEEE, 2011, pp. 1-8.

[17] E. Biasotto, M. Oleskovicz, D. V. Coury, and C. J. dos Santos, "Proposal for a New Structural and Electrical Crossarm Standard for $15 \mathrm{kV}$ Distribution Networks," J. Control. Autom. Electr. Syst., vol. 25, no. 4, pp. 516-526, 2014.
[18] Autodesk Inc., "Autodesk Simulation Mechanical," San Rafael, California, U.S. [En Línea] Disponibe en: $\mathrm{http}: / / \mathrm{www}$. autodesk.com/products/simulationmechanical/overview.

[19] Creative Pultrusions INC., "The Pultex Pultrusion Design Manual," 2015. [En Línea] Disponible en: http://www.creativepultrusions.com/productssolutions/fabrication-capabilities/pultexc2ae-pultrusiondesign-manual1/ 